\title{
Diagnostic Cardiac Catheterization for Congenital Heart Disease in Nepal
}

Regmi $S^{*}$, Maskey $A^{*}$, Dubey $L^{*}$, Malla $R^{*}$, Bhatt $Y^{*}$, Rajbhandari $R^{*}$, Limbu $Y R^{*}$, $\mathrm{KC} \mathrm{MB}$ *

*Shahid Gangalal National Heart Centre, Bansbari, Kathmandu

\section{ABSTRACT}

Accurate preoperative anatomic and functional diagnosis is of vital importance for surgical repair of congenital heart disease. The diagnostic accuracy of echocardiography alone in congenital heart disease is satisfactory but interventional catheterization is still required to derive the adequate information before surgical repair. This is a retrospective review of our early results with cardiac catheterization for congenital heart disease in Shahid Gangalal National Heart Center (SGNHC), Nepal.

Correspondence:

Dr Shyam Regmi

Department of cardiology

Shahid gangalal National Heart Center

Kathmandu, Nepal 


\section{INTRODUCTION}

Accurate preoperative anatomic and functional diagnosis is of vital importance for surgical repair of congenital heart disease $(\mathrm{CHD})^{1}$. Cardiac catheterization and angiocardiography made it possible to derive adequate information for cardiac repair. Cardiac catheterization carries a risk especially in infants and in neonates. Recent technical advances in echocardiography provide the adequate information, even in the complex lesions, needed for cardiac surgery in many patients without invasive catheterization ${ }^{2}$. So question arise whether catheterization is necessary before cardiac surgery in our setting where both echocardiography as well as catheterization are in the developing stages. This is a retrospective review of our early results with cardiac catheterization for congenital heart disease in SGNHC, Nepal.

\section{PATIENTS AND METHODS}

All children with CHD who underwent diagnostic cardiac catheterization between August 2003 and February 2010 were included and data was analyzed retrospectively. The need for cardiac catheterization was primarily determined by a cardiologist or suggested by a cardiac surgeon. Patient were initially evaluated by complete history, physical examination, chest X-ray, ECG and detailed echocardiographic examination. Before the procedure, a detailed explanation of purpose, route and possible complications were discussed with the patient party. Both written and informed consent were taken.

Most of the cases were done under general anesthesia. Local anesthesia was used in older patients. Complete hemodynamic assessment with pressures, pulmonary vascular resistance, oxygen saturations and blood gas analysis as well as angiographic assessment were performed.

\section{RESULTS}

During August 2003 to February 2010, we performed 1143 diagnostic paediatric cardiac catheterizations. Age ranges were from few weeks to 16 years. The most common indications for diagnostic catheterization was delineation of pulmonary artery anatomy, origin and course of coronaries, presence of significant sized MAPCAs in tetralogy of fallot (TOF) in 405 (35.43\%) and hemodynamic assessment for operability in patient with ventricular septal defect with severe pulmonary hypertension in 229 (20.03\%). Other indications includes atrial septal defect with severe pulmonary artery hypertension (PAH) 154 (13.47\%), PDA with severe PAH 47 $(4.11 \%)$, primary pulmonary hypertension 35

Table: Diagnosis of the patients who underwent diagnostic catheterization:

\begin{tabular}{lcl}
\multicolumn{1}{c}{ Diagnosis } & Number of patients & Percentage \\
TOF & 405 & 35.43 \\
VSD with severe PAH & 229 & 20.03 \\
ASD with severe PAH & 154 & 13.47 \\
PDA with severe PAH & 47 & 4.11 \\
Tricuspid atresia & 29 & 2.54 \\
Truncus arteriosus & 7 & 0.67 \\
TGA & 41 & 3.58 \\
Ebstein's anomaly & 3 & 0.26 \\
AV canal defect with severe PAH & 24 & 2.11 \\
DORV & 65 & 5.69 \\
TOF with pulmonary atresia & 26 & 2.27 \\
Congenitally corrected TGA & 8 & 0.67 \\
Coarctation of aorta & 19 & 1.66 \\
Primary pulmonary hypertension & 35 & 3.06 \\
Others & 51 & 4.46
\end{tabular}


Six (0.53\%) patients died immediately after procedure, $11(0.96 \%)$ developed acute limb ischemia due to thrombosis at puncture site.

Among the 405 TOF patients, right sided aortic arch was found in $142(35.06 \%)$, anomalous coronaries in 119 (29.38\%), significant sized MAPCAs in 222 (54.81\%), inadequate PA size for intracardiac repair was found in123 (30.37\%),

(3.06\%), tricuspid atresia $29(2.54 \%)$, truncus arteriosus $7(0.67 \%)$, transposition of great arteries $41(3.58 \%)$, Ebstein's anomaly $3(0.26 \%), A V$ canal defect with severe PAH $24(2.11 \%)$, DORV $65(5.69 \%)$, TOF with pulmonary atresia 26 $(2.27 \%)$, congenitally corrected TGA $8(0.67 \%)$, two or more shunt anomalies $43(3.77 \%)$, coarctation of aorta $19(1.66 \%)$, total anomalous pulmonary venous drainage $6(0.58 \%)$ and double outlet left ventricle $2(0.17 \%)$.

\section{DISCUSSION}

Congenital heart disease is the single most important major congenital defect with prevalence of 8-10 per 1000 and is responsible for much mortality and morbidity in infants and children ${ }^{3}$. Cardiac catheterization has proved its value as a major tool in the diagnosis of congenital cardiac defects $^{4}$.

Some literatures demonstrated no advantage of cardiac catheterization over echocardiography in simple shunt lesions such as ASD, VSD and PDA 2,5 . However, there is controversy over whether cardiac catheterization is routinely indicated for preoperative diagnosis of more complex lesions.
Echocardiography alone may be insufficient to obtain the anatomic and functional information needed for surgical repair in complex congenital heart disease and may require preoperative catheterization. We performed diagnostic cardiac catheterization in 1143 patients with congenital cardiac defects with low periprocedural mortality rate.

Indications for catheterization were mainly delineation of pulmonary artery anatomy in patients with TOF, calculations of Qp/Qs and PVR in patients with left to right shunts with severe $\mathrm{PAH}$ to check for operability and delineation of anatomy in other complex CHD. These indications are not different from those reported by others. Catheterization is commonly indicated for hemodynamic assessment as it is not yet possible to measure accurately pulmonary artery pressure, Qp/Qs and PVR by echo.

In conclusion, though diagnostic accuracy of echocardiography for safe cardiac surgery is increasing, cardiac catheterization is necessary to delineate pulmonary artery size, coronary anatomy, presence of significant MAPCAs and hemodynamic assessment for operability.

\section{REFERENCES}

1. Stark J, Smallhorn J, Huhta J et al. Surgery for congenital heart defects diagnosed with cross-sectional echocardiography. Circulation 1983; 68: 129-38

2. Krabill KA, Ring WS, Foker JE et al. Echocardiographic versus cardiac catheterization diagnosis of infants with congenital heart disease requiring cardiac surgery. Am J Cardiol 1987; 60: 351-54

3. Zabal C, Lince R, Buendia A, Attie F, Rios
MA. Interventional cardiology in congenital heart disease. Arch Inst Cardiol Mex 1999; 69: 63-68

4. Inglessis I, Landzberg MJ. Interventional catheterization in adult congenital heart disease. Circulation 2007; 115: 1622-33

5. Freed MD, Nadas As, Norwood WI, Castaneda AR. Is routine preoperative cardiac catheterization necessary before repair of secundum and sinus venosus atrial septal defects? JACC 1984; 4: 333-36 